\title{
CARACTERIZACIÓN DEL CULTIVO DE BALSA (Ochroma pyramidale) EN LA PROVINCIA DE LOS RÍOS - ECUADOR
}

\author{
${ }^{\bullet}$ Betty González Osorio ${ }^{1,2}$, Ximena Cervantes Molina ${ }^{1}$, Emma Torres Navarrete ${ }^{1,3}$, Carlos Sánchez Fonseca ${ }^{1}$ Luis Simba $^{4}$ \\ ${ }^{1}$ Unidad de Investigación Cientifica y Tecnológica, Universidad Técnica Estatal de Quevedo, km 7 vía \\ Quevedo - El Empalme, C.P.73. Mocache, Los Ríos, Ecuador. \\ ${ }^{2}$ Facultad de Ciencias Empresariales, Universidad Técnica Estatal de Quevedo, $\mathrm{km} 1$ 11/2 vía \\ Quevedo - Santo Domingo de los Tsáchilas, Quevedo,Los Ríos, Ecuador •bettygon75@yahoo.com \\ ${ }^{3}$ Facultad de Ciencias Pecuarias, Universidad Técnica Estatal de Quevedo, $\mathrm{km} 7$ vía a El Empalme, \\ Mocache, Los Ríos, Ecuador \\ ${ }^{4}$ Facultad de Ciencias Agrarias, Universidad Técnica Estatal de Quevedo, $\mathrm{km} 1$ 1/2 vía \\ Quevedo- Santo Domingo de los Tsáchilas, C.P.73. Quevedo, Los Ríos, Ecuador.
}

RESUMEN

Qe realizó la caracterización del cultivo de Balsa (Ochoroma pyramidale), en la provincia de Los Ríos , Ecuador, durante el año 2008. Se determinó el establecimiento, manejo y comercialización de esta especie. Además se establecieron costos, ingresos, rentabilidad y punto de equilibrio del cultivo mediante encuestas a productores de balsa. Los resultados demuestran que los productores utilizan la semilla nativa procedente de Ecuador. El 53.50\% compran las plántulas en viveros de la localidad mientras que el $46.50 \%$ realizan su propio vivero. Cabe destacar que los productores realizan labores de establecimiento y mantenimiento a sus cultivos, no obstante el $94.00 \%$ de ellos no reciben asistencia técnica, lo cual incide en la calidad de las plantaciones y su rendimiento. Se estableció que las superficies sembradas van desde 0.37 hasta 44.40 hectáreas; donde $34.25 \%$ pertenecen a plantaciones de un año; $32.54 \%$ son plantaciones de tres años y en menores porcentajes se encuentran cultivos de dos, cuatro y cinco años. Cuando han alcanzado una edad de tres a cuatro años, los árboles son comprados directamente en las fincas de los productores en forma de trozas y por camionada, por empresas de la localidad como son: PLANTABAL, INMAIA y BALSAFLEX, las mismas que exportan balsa procesada a mercados de Europa, Asia y Norteamérica. Los costos totales en cuatro años de producción en una hectárea de balsa ascienden a 2,477.06 dólares con ingresos de 6,000.00 dólares, obteniendo un beneficio neto de 3,522.94 dólares una rentabilidad de 142.00 por ciento y el punto de equilibrio en unidades físicas $22.80 \mathrm{~m}^{3}$ de balsa y 912.10 dólares en unidades monetarias por hectárea.

Palabras claves: balsa, viveros, plántulas, costo de producción, rentabilidad, punto de equilibrio
Abstract

A characterization of balsa (Ochoroma pyramidale) Acrop was made in the Los Ríos province Ecuador, during the 2008. The establishment, manage and commercialization of this specie was determined. Also the costs of production, incomes, profitability and the equilibrium point of this crop were determined through surveys made to the balsa planters. It was determined that producers use native seeds proceeding from Ecuador. $53.50 \%$ of the surveyed planters get their seedlings from local plant nursering while $46.50 \%$ get their seedlings from their own plant nursering. It is important to mention that planters do the establishment and maintenance labors of their crops by theirself, although $94.00 \%$ of them never has any technical assistance which is determining for the quality and yield of the crops. It was determined that the surface used to establish this crop oscilate from 0.37 to 44.40 hectares where $34.25 \%$ belongs to one year plantations, $32.54 \%$ to three years, and with the lower percentage crops of two, four and five years can be found. When the trees have reach an average age of three to four years they are directly bought at the planters' farm cutted in to pieces and transported by trucks by local enterprises as PLANTABAL, INMAIA and BALSAFLEX, whom process the balsa wood and export to the European, Asiatic and American markets. The total production costs at four years, of an hectare of balsa can get as high as \$2,477.06 USD with incomes of \$6,000.00 USD getting \$3,522.94 USD as net benefit with a profitability of $142.00 \%$ and an equilibrium point of $22.80 \mathrm{~m}^{3}$ of physic units of balsa wood and $\$ 912.00$ USD in monetary units per hectare.

Key words: balsa wood, greenhouse, seedlings, costs of production, profitability, equilibrium point.

\section{INTRODUCCIÓN}

$\mathrm{O}$ chroma pyramidale, también llamada balsa, es una especie forestal y maderera que posee gran demanda en el mercado internacional. Se cultiva de manera natural y por reforestación, especialmente en la selva sub-tropical de Ecuador, donde es uno de los recursos forestales y maderables de mayor aprovechamiento; por tal razón es uno de los rubros económicos de

Recibido: Mayo, 2010. Aceptado: Julio, 2010.

Publicado como NOTA TECNICA en Ciencia y Tecnología 3(2): 7-11. 2010 importancia en la economía de nuestro país. En el comercio internacional se conoce por su nombre común de balso ecuatoriano. La especie ha alcanzado un alto nivel de desarrollo, desde su reforestación hasta su posterior transformación, convirtiéndola en la madera de balsa de mayor calidad a nivel mundial. La especie es de gran importancia comercial en la cuenca del Río Guayas en Ecuador; de donde se obtiene el 95\% de la cosecha mundial (Butterfield, 1995). Hoy en día, la madera se usa para modelos, artesanías y juguetes, 
como chapa de interiores en construcciones en capas con material sintético, aluminio y madera, en donde se necesite fortaleza y propiedades aislantes. Se usa también como material aislante masivo y libre de fuerzas electrostáticas en barcos para transporte criogénico (Chudnoff, 1984; Villavelez, 1979). A pesar de poseer fibras cortas al igual que la mayoría de otras especies de madera dura, la madera de balsa se ha usado de manera limitada para la producción de pulpa y papel (Hueck, 1961; Webb et al., 1984).

En la actualidad, Ecuador posee, más de 20 mil hectáreas de plantaciones entre bosques naturales y reforestados. Siendo las zonas de mayor producción las provincias del Guayas, El Oro, Los Ríos y Pichincha. Las exportaciones se realizan principalmente siguiendo la demanda externa ya que la demanda nacional es muy pequeña. En nuestro país apenas 10 por ciento es utilizado para elaborar artesanías caseras, mientras que el 90 por ciento se exporta principalmente a Estados Unidos y Comunidad Económica Europea en forma de tableros, láminas, bloques y madera aserrada (Obregón, 2005; Vocalia,2007).

La balsa requiere de un clima cálido y húmedo. La cantidad mínima de precipitación que tolera es de alrededor de $1500 \mathrm{~mm}$ anuales (Marshall, 1939), excepto a lo largo de corrientes de agua, en donde el nivel del agua subterránea se encuentra cerca de la superficie y puede ser absorbida por las raíces (Whitmore et al., 1983); además esta especie demanda una rica provisión de nutrientes (Fors, 1965) y un suelo bien drenado (León et al., sf). De hecho, se reporta que los árboles de balsa mueren con facilidad debido a las inundaciones (Streets, 1962).

La balsa coloniza suelos arcillosos, margosos y limosos, e incluso el relleno de construcción recientemente depositado, pero no tolera los suelos de alta salinidad (Betancourt, 1968). Las características edafoclimáticas de la provincia de Los Ríos, hacen de este territorio, un espacio idóneo para la producción de esta especie (crecimiento, mejores características de su madera, posición ante los mercados). No obstante, todo este potencial lo ha liderado un limitado número de empresas reforestadoras, dejando a pequeños agricultores fuera de esta actividad (Espinoza, 2007).

El objetivo de esta investigación fue evaluar el ciclo productivo de la balsa (Ochoroma pyramidale) en la provincia de Los Ríos.

\section{Materiales y Métodos}

$\mathrm{L}^{2}$ a investigación se realizó en la provincia de Los Ríos, localizada en una zona climática denominada Tropical manzónico; con un clima Tropical seco y con una temperatura media que oscila entre 22 y $33^{\circ} \mathrm{C}$, en la época seca es fresco y en época de lluvias es caluroso, con altas y continuas precipitaciones.

Para recabar información general del cultivo, así como costos de producción, precios, volúmenes de venta y sistemas de comercialización se diseñó un formulario de preguntas, el mismo fue aplicado a productores que tenían plantaciones de balsa en la provincia de Los Ríos. Para realizar la investigación propuesta se consideró como población 86 familias campesinas que siembran balsa en la provincia de Los Ríos, la información correspondiente se la obtuvo a través de un censo, esta técnica permitió abarcar a todos los involucrados en el cultivo de balsa.

Los resultados fueron debidamente codificados, tabulados y analizados estadísticamente. La información que se obtuvo fue resumida en cuadros y figuras, con lo cual se cubrieron los aspectos tratados en los formularios $\mathrm{y}$, en las variables planteadas para la evaluación del estudio.

Para caracterizar el cultivo de la balsa, los aspectos evaluados en las encuestas fueron:

- Características generales de la finca (ubicación, explotación, características del suelo, tierras labradas y cultivos)

- Modalidades tecnológicas (riego, maquinaria, hábitat y red viaria)

- Asistencia técnica

- Mano de obra

- Financiamiento

- Precios

- Acceso a mercados

- Aspectos culturales

- Evaluación económica (costos, ingresos y rentabilidad)

En cuanto a la evaluación económica se consideró la fase de producción que tiene la balsa, considerando una edad de cuatro años para realizar el último aprovechamiento. Los costos se establecieron durante el tiempo de vida útil de la plantación, se clasificaron los costos en fijos y variables. Entre los costos que se consideraron fueron: alquiler de terreno, preparación de suelo, siembra, control de malezas, control fitosanitario, fertilización, cosecha, mano de obra indirecta, costos de oportunidad (tasa pasiva se la consideró en un $4.56 \%$ anual). Referente a los costos e ingresos se calcularon con precios referenciales del año 2008. La venta de balsa se consideró en metros cúbicos de madera rolliza, la rentabilidad se la obtuvo mediante la relación beneficio-costo y para conocer el nivel mínimo que se debe de producir para no perder ni ganar en una hectárea de balsa, se aplicó la ecuación del punto de equilibrio. 


\section{Resultados}

\section{Características generales Superficies sembradas}

$\mathrm{E}^{\mathrm{n}}$ la provincia de Los Ríos existen áreas cultivadas que van desde 0.37 hasta 44.40 hectáreas de balsa con una media de 3.71 ha y una desviación típica de 6.16. El mayor porcentaje de productores tienen plantaciones de un año $(34.25 \%)$, plantaciones con tres años representan el $32.54 \%$ de la población estudiada, el $23.76 \%$ corresponde a plantaciones de dos años, en tanto que las plantaciones de cuatro y cinco años, que ya están listas para ser aprovechadas representan el 7,39\% y $2,06 \%$ respectivamente

\section{Manejo del cultivo Selección del material vegetativo}

El sistema de propagación de la balsa es solo sexual (semillas) siendo el único método conocido y recomendado para plantaciones de esta especie. El $98.84 \%$ de los productores evaluados por el estudio utilizan semillas nativas de Ecuador. Para establecer este cultivo, un $53.50 \%$ de los productores compran las plántulas en viveros de la localidad y un $46.50 \%$ realizan su propio vivero.

\section{Actividades de presiembra}

Para establecer sus plantaciones el $73.26 \%$ de los productores realizan roza manual, ya que el terreno debe de estar limpio totalmente de malezas. La delineación y trazado del cultivo es otra actividad que el agricultor realiza, donde las distancias entre filas y plantas deben ir de acuerdo a la topografía del terreno, clima y mano de obra; el $62.79 \%$ de los productores utilizan el sistema de siembra en marco real.

\section{Siembra}

La densidad de siembra es variable, el $27.91 \%$ de los productores siembran las plántulas de balsa a una densidad de $3 \times 3 \mathrm{~m}$ (1,100 plantas); $18.60 \%$ siembran a 4 x 4 m (625 plantas); $13.95 \%$ siembran a 3 x 4 m (833 plantas) y el $39.51 \%$ siembran en otras densidades.

\section{Labores culturales}

Con el objetivo de evitar la competencia por los nutrientes, agua del suelo y sol, entre las plantas de balsa y malezas, es importante tener limpia la plantación, es así que el $53.49 \%$ de los productores de la provincia de Los Ríos realizan el control manual, mientras el $46.51 \%$ aplica productos químicos.

\section{Asistencia Técnica}

La asistencia técnica que se le aplica al cultivo de balsa es indispensable para que su desarrollo sea idóneo, no obstante el $94 \%$ de los pequeños y medianos productores de esta especie en la provincia de Los Ríos no reciben esta capacitación y únicamente el $6 \%$ la adquieren a través de técnicos de empresas comerciales.

\section{Comercialización}

En nuestro país existen dos grupos de productores: las industrias, que además de producir, fabrican o procesan balsa en productos terminados; y los campesinos, que la cultivan y venden en pie directamente en las fincas a los intermediarios (Figura 1). En la provincia de Los Ríos las empresas BALSAFLEX,

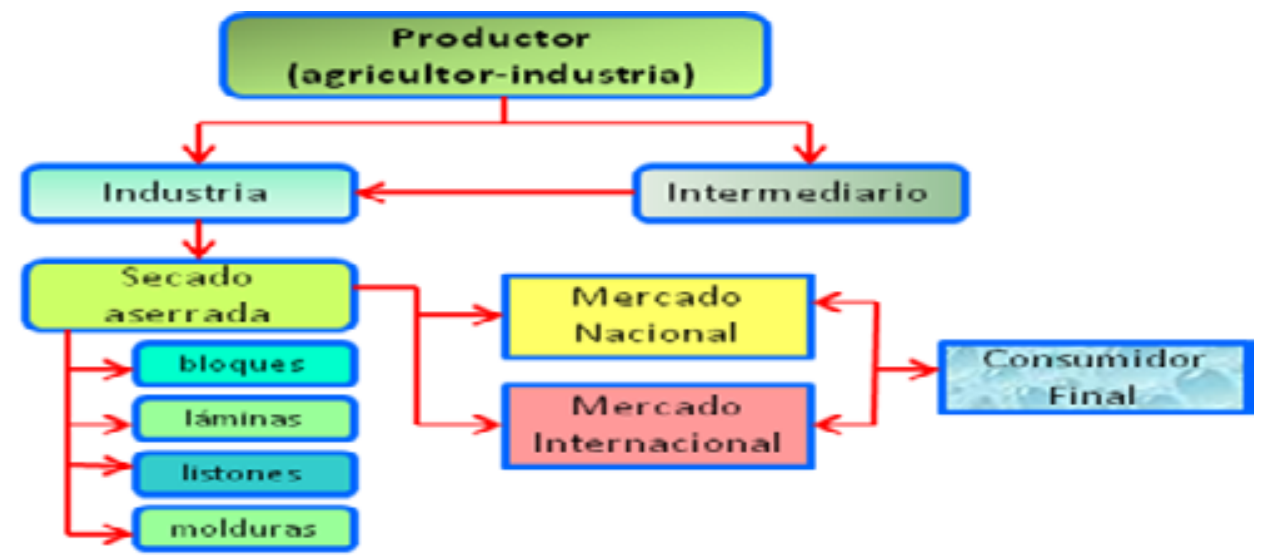

Figura 1. Cadena de comercialización de la balsa en la Provincia de los Ríos 
INMAIA y PLANTABAL exportan la balsa en forma de bloques y madera aserrada teniendo gran demanda en los mercados de Europa, Asia y Norteamérica.

La maduración económica y física de la balsa tiene lugar a una edad temprana. Los árboles de crecimiento rápido producen el mejor rendimiento y el mejor producto cuando tienen de cuatro a seis años de edad (Longwood, 1962). Una vez que las plantas tienen cuatro años estas son cortadas por el campesino y según la demanda del mercado, el diámetro preciso va de 13,20 y $27 \mathrm{~cm}$, no obstante las industrias requieren un diámetro más grande que va de 33 a $40 \mathrm{~cm}$ y árboles que tengan de cuatro a seis años de edad. Los árboles son comprados directamente en las fincas de los campesinos en trozas y por camionada (Nissan) el precio oscila entre 90.00 a 110.00 dólares o un carro bananero $1,000.00$ a 1,100.00 dólares.

\section{Evaluación económica}

En lo referente a la estructura del costo total se observa que los más representativos son las labores de mantenimiento con 50.46\%; seguido del alquiler de terreno con un $20.19 \%$ y el establecimiento del cultivo con el 19.94\% (Figura 2).

Los costos variables promedios de una hectárea de balsa son de 1845.43 dólares, mientras que los costos fijos promedios ascienden a 631.63 dólares, siendo el costo total de producción promedio de 2477.06 dólares; con producciones promedios de $150 \mathrm{~m}^{3} \mathrm{ha}^{-1}$, a un precio de venta promedio de 40.00 dólares $/ \mathrm{m}^{3}$, se tienen ingresos brutos de 6000.00 dólares, dando como utilidad 3522.94 dólares y una relación Beneficio Costo 1.42 dólares.

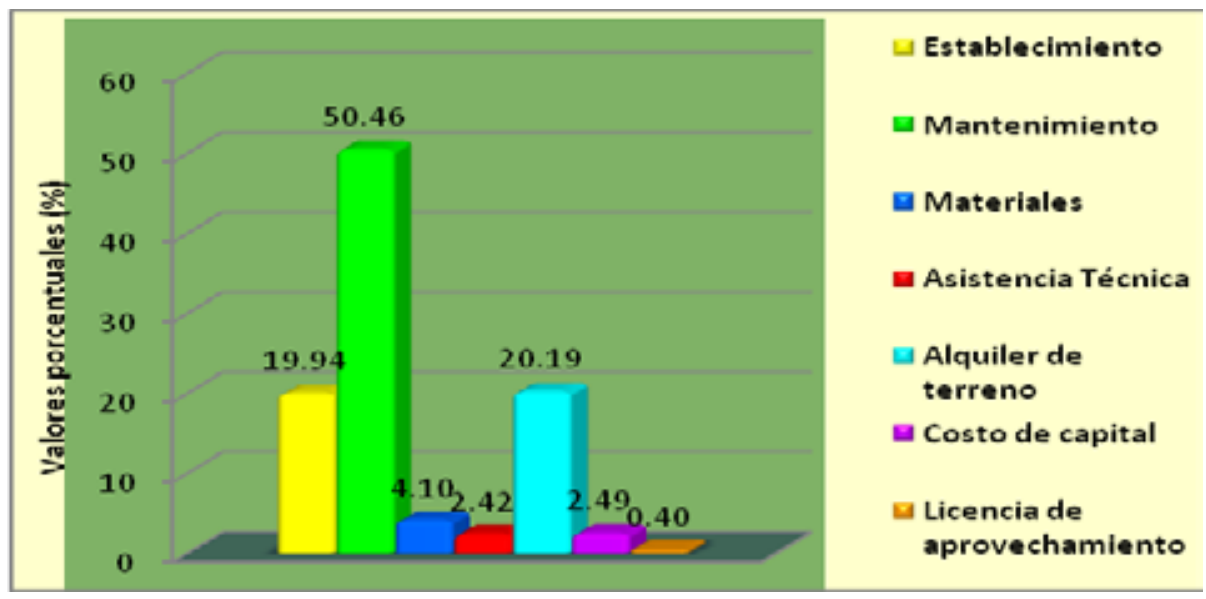

Figura 2. Costos de producción durante cuatro años en una hectárea de balsa en la provincia de Los Ríos, 2008

El punto de equilibrio en unidades físicas resultó $22.80 \mathrm{~m}^{3}$ de balsa y 912.10 USD de unidades monetarias por hectárea, es decir que para que el productor de la provincia de Los Ríos no pierda ni gane debe estar dentro de dicho nivel de producción (Figura 3).

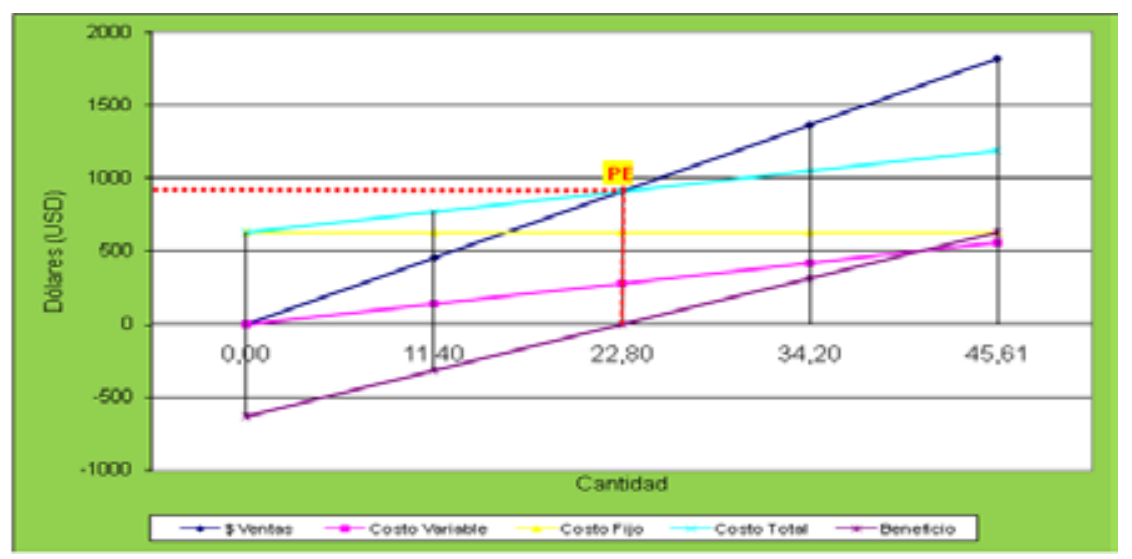

Figura 3. Punto de equilibrio físico y monetario durante cuatro años en una hectárea del cultivo de balsa en la provincia de Los Ríos, 2008 


\section{Conclusiones}

T os productores de balsa utilizan semilla procedente de Ecuador.

Para el establecimiento de las plantaciones, el $53.50 \%$ de los productores de balsa compra plántulas en viveros de la localidad y el $46.50 \%$ realiza su propio vivero.

En la provincia de Los Ríos, los pequeños y medianos productores de balsa poseen superficies embradas que van desde 0.37 hasta 44.40 hectáreas.

Según las encuestas las plantaciones con mayor área son aquellas de un año de edad (35,25).El 94.00\% de los productores de balsa no reciben asistencia técnica para el manejo de sus plantaciones.

Los costos más representativos en el cultivo de balsa son las labores de mantenimiento.

El costo total de producción del cultivo de balsa asciende a 2,477.06 USD con ingresos de 6,000.00 dólares obteniendo un beneficio neto de 3,522.94 dólares y una rentabilidad de $142.00 \%$.

El punto de equilibrio en unidades físicas es de $22.80 \mathrm{~m}^{3}$ de balsa y 912.10 USD de unidades monetarias por hectárea

\section{Literatura Citada}

Betancourt, S. 1968. Monografía de la balsa o lanero. Técnica Forestal 3. Bogotá, Colombia: Instituto Nacional de Desarrollo y Aprovechamiento Forestales. 7 p.

Butterfield, R. 1995. Desarrollo de especies forestales en tierras bajas húmedas de Costa Rica. Turrialba, Costa Rica, CATIE, Serie Técnica, Informe Técnico No 260,41p.

Chudnoff, M. 1984. Tropical timbers of the world. Agric. Handb. 607. Washington, DC: U.S. Department of Agriculture. 464p.
Espinoza, E. 2007. Incentivan cultivo de Balsa. (En línea). Consultado 12 Oct. 2009. Disponible

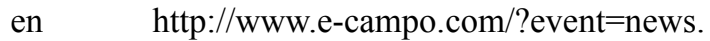
d is p la y \& i d = A 3662695 - 188 B - 7 C 0 F A69FDD2F484F46

Fors, A. 1965. Maderas cubanas. Habana, Cuba: Instituto Nacional de Reforma Agrícola. 162 p.

Hueck, K. 1961. The forests of Venezuela. Heft 14. Hamburg, Germany: Verlag Paul Parey. 106p.

León, R; Muñoz, L. (s.f.) Maderas colombianas. Bogotá, Colombia: Fondo de Promoción de Exportaciones. $117 \mathrm{p}$.

Longwood, F. 1962. Present and potential commercial timbers of the Caribbean. Agric. Handb. 207. Washington, DC: U.S. Department of Agriculture. $167 \mathrm{p}$.

Marshall, R.C. 1939. Silviculture of the trees of Trinidad an Tobago, British West Indies. London: Oxford University Press. 247 p.

Obregón, C. 2005. La Balsa una especie con futuro. (en línea). Consultado 10 Oct. 2009. Disponible en http://www.revista-mm.com/rev54/especie.pdf

Streets, R.1962. Exotic forest trees in the British Commonwealth. Oxford, England: Clarendon Press. $750 \mathrm{p}$.

Vocalia. 2007. Cultivo de la Balsa. (en línea). Consultado 13 Oct.2009. Disponible en http://www.fdacm. com/aero/artic/articulo.asp? $\mathrm{id}=28$

Villavelez, V.; Meniado, J. 1979. Notes on balsa (Ochroma pyramidale Cav.). Forpride Digest. 8(3/4):25-30.

Witmore, C.; Wooi-Khoon, G. 1983. Growth analysis of the seedlings of balsa, Ochroma lagopus. New Phytologist.95:305-311

Webb, B.; Wood, J.; Smith, P.; Henman, G. Sian. 1984. A guide to species selection for tropical and subtropical plantations. Tropical Forestry Papers 15. Oxford, England: Commonwealth Forestry Institute, University of Oxford.256 p. 